\title{
The morphological growth patterns of colorectal liver metastases are prognostic for overall survival
}

\author{
Kåre Nielsen ${ }^{1}$, Hans C Rolff ${ }^{2,3}$, Rikke L Eefsen ${ }^{3,4}$ and Ben Vainer ${ }^{1}$ \\ ${ }^{1}$ Department of Pathology, Rigshospitalet, Copenhagen University Hospital, Copenhagen, Denmark; \\ ${ }^{2}$ Department of Surgical Gastroenterology, Rigshospitalet, Copenhagen University Hospital, Copenhagen, \\ Denmark; ${ }^{3}$ The Finsen Laboratory, Rigshospitalet, Copenhagen University Hospital, Copenhagen, Denmark \\ and ${ }^{4}$ Department of Oncology, Rigshospitalet, Copenhagen University Hospital, Copenhagen, Denmark
}

\begin{abstract}
Colorectal metastases in the liver grow according to three histological patterns: a pushing pattern, a replacement pattern, and a desmoplastic pattern. The objective of the current study was to explore the prognostic significance of these three growth patterns for survival. The study included 217 consecutive patients, liver resected between 2007 and 2011 due to hepatic metastases from colorectal adenocarcinoma. The growth patterns were assessed on archival hematoxylin and eosin-stained tissue sections. In 150 metastases, the density of the immune cell infiltrate at the tumor periphery was judged by a semi-quantitative method. The prevalence of the pushing-type, the desmoplastic-type, and the replacement-type was $33 \%, 32 \%$, and $11 \%$, respectively; $24 \%$ of the metastases displayed a mixed pattern. Kaplan-Meier analysis and Cox regression demonstrated a prognostic significance of the growth patterns $(P=0.0006$, log-rank test), as the replacement pattern appeared as an independent predictor of poor overall survival. For patients with replacement growth, the hazard of death was 2-2.5 times higher than for patients with pushing growth $(P=0.004$, cox regression) or mixed growth $(P=0.01)$, and nearly four times higher than for patients with desmoplastic growth $(P<0.0001)$. The negative prognostic effect of the replacement growth pattern was even more pronounced after adjusting for tumor size. Desmoplastic growth corresponded with small tumor size, dense lymphocytic infiltration and a more favorable prognosis. Eventually, the growth patterns may contribute to a histology-based prognostic biomarker for patients with colorectal liver metastases.

Modern Pathology (2014) 27, 1641-1648; doi:10.1038/modpathol.2014.4; published online 23 May 2014
\end{abstract}

Keywords: colorectal cancer; growth pattern; histopathology; liver; metastasis; prognosis; survival

During the last 5-10 years, major advances have been made in both the medical and surgical treatment of liver metastases from colorectal carcinoma. The number of patients who undergo liver resection with curative intent has therefore increased significantly. However, so far, little is known on the biological, clinical, and histopathological features that determine prognosis after hepatic resection. The revised and more active treatment strategy has thus fostered a demand for new and better prognostic and predictive markers that can be

Correspondence: K Nielsen, Department of Pathology, Rigshospitalet, Copenhagen University Hospital, Frederik V's Vej 11, 2100 Copenhagen, Denmark.

E-mail: kaare.nielsen@regionh.dk

Received 2 October 2013; accepted 20 December 2013; published online 23 May 2014 used for selecting the right treatment regimen for each patient. For primary colorectal cancer, the malignant transformation from adenoma to carcinoma is by now very well described; far less described are the events that govern the progression of metastases in the liver. A greater understanding of the diverse infiltration mechanisms of the metastases, including the intricate interaction with the hepatic microenvironment, might lead to new prognostic and therapeutic prospects.

Vermeulen et $a l^{1}$ identified three histological growth patterns for colorectal liver metastases, suggesting biological differences: (i) a pushing growth pattern, in which the metastasis expands by compression of the surrounding liver parenchyma, pushing the liver cell plates aside; (ii) a desmoplastic growth pattern, in which a fibrous rim separates the metastasis from the liver paren- 

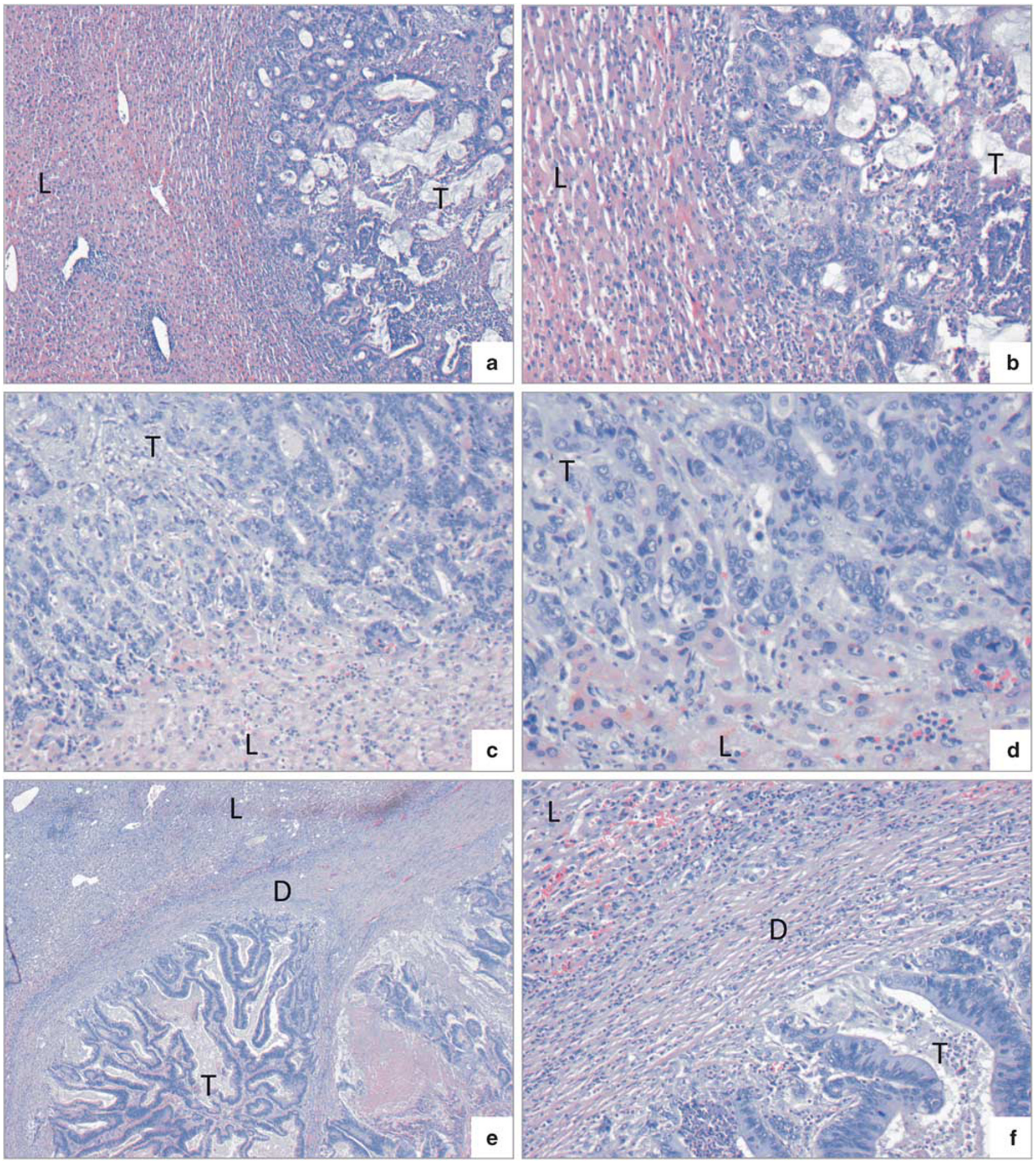

Figure 1 Microphotographs of colorectal adenocarcinoma liver metastases (hematoxylin and eosin-stained sections; T, tumor; L, liver parenchyma; D, desmoplastic rim). (a and b) show a metastasis of the pushing-type. The metastasis grows by compression of the liver parenchyma. Along the edge of the tumor, liver cell plates are strongly narrowed and run in parallel with the tumor invasion front. In (c and d), a metastasis with replacement growth is depicted. (c) shows how the tumor tissue preserves the architecture of the hepatic tissue. The reticulin framework of the liver tissue is conserved in the peripheral 10-20 cell rows of the tumor. (d) shows the intimate cell-cell contacts between tumor cells and hepatocytes. In (e and $\mathbf{f}$ ) a metastasis is depicted with a desmoplastic growth pattern. The metastasis is separated from the surrounding liver parenchyma by desmoplastic stroma. Typically, the desmoplastic rim presents as two compartments: a dense part toward the tumor and an edematous part toward the liver tissue harboring an intense immune cell infiltrate.

chyma; and (iii) a replacement growth pattern, in which tumor cells infiltrate the liver cell plates, replacing the hepatocytes while co-opting hepatic stroma and sinusoids. Examples of the three types of expansion are included in Figure 1. The growth patterns differ with respect to angiogenesis, as 
replacement-type metastases exert virtually no angiogenic properties at the invasive front. ${ }^{1-4}$ The desmoplastic growth pattern is consistent with high proteolytic activity. ${ }^{4,5}$

Although the morphological categorization described by Vermeulen et $a l^{3}$ has been recognized in various studies, the prognostic significance remains controversial. Van den Eynden et $a l^{3}$ identified pushing growth as an independent predictor of poor 2-year survival, whereas Nagashima et al found a detrimental effect of an infiltrative tumor margin similar to the replacement pattern. Several studies comparing metastases with and without desmoplastic encapsulation have reported a positive impact on survival of desmoplasia per se. ${ }^{7-13}$ In consequence, the aim of the present study was in a population treated according to modern guidelines and indications to examine the prognostic value of the histological growth patterns for survival.

\section{Materials and methods}

\section{Patients}

The study included 217 consecutive patients (134 men and 83 women) diagnosed with colorectal adenocarcinoma liver metastases. The patients underwent hepatic resection with curative intent at Rigshospitalet, Copenhagen University Hospital, during the period from January 2007 to August 2011. Median age of the patients was 65 years (range: 19-92). The growth pattern of the metastases was assessed on archival $4 \mu \mathrm{m}$ hematoxylin and eosin-stained tissue sections cut from formalin-fixed paraffin-embedded specimens from the tumor-liver boundary. Patients were excluded if no tissue sections complying with these criteria and of at least $1 \mathrm{~cm}^{2}$ were available. In addition, patients whose metastases appeared with a positive margin or with heat-induced damage of the invasion front (due to the surgical procedure or previous radio frequency ablation) were excluded. For each patient, only the tissue section with the highest representation of the interface was examined, in order to standardize the material, recognizing that it is impossible to assess the entire surface of a sphere in a retrospective study.

In addition to this, the material from 22 re-resections of 16 patients with recurrent colorectal liver metastases was evaluated histologically in order to compare the growth pattern across repeat hepatic resections. In the survival analysis, these patients were included by the growth pattern of their first metastasis.

Survival data were acquired from the National Patient Index. The largest diameter of the metastases as well as the number of metastases per patient were obtained from the original pathology reports. Oncological treatment data on the patients were not available. All procedures followed were in accordance with the ethical standards of the national committee on human research and with the Helsinki Declaration of 1975, as revised in 2008. The study was approved by the Regional Scientific Ethics Committee (reference no. H-2-2011-121).

\section{Histological Assessment}

The histological growth pattern of the metastases was characterized as 'pushing-type', 'desmoplastictype' or 'replacement-type', according to the procedure described by Vermeulen et al. ${ }^{1}$ In order to purify these three categories, a definition for metastases with multiple growth patterns was demanded. Hence, a 'mixed-growth pattern' was defined as a morphology where at least two growth patterns were present, both appearing in at least $20 \%$ of the tumor periphery. Mixed-type metastases were included with a dominating primary growth pattern and one or two secondary growth patterns. Metastases with mixed-type margins have been reported earlier, but guidelines for cut-off point and categorization have not yet been published. ${ }^{1-4}$ In previous studies, little intra- and inter-observer variability was found for Vermeulen's procedure.1,2,4 After consensus readings by two observers ( $\mathrm{KN}$ and $\mathrm{BV}$ ) of 20 cases, one observer (KN) assessed all sections. Cases of doubt were judged by consensus review. The assessment was blinded with regard to prognosis and other clinical conditions.

In 150 patients, the density of the lymphocytic infiltrate at the tumor edge was scored on a semiquantitative scale from I to III, where a score of I indicated no or mild immune cell infiltration with a diffuse distribution in the tissue; II indicated moderate immune cell infiltration, mainly focal and with no appreciable tissue damage; and III indicated intense immune cell infiltration, continuous and with marked tissue destruction.

\section{Statistical Analysis}

The data were analyzed for statistical significance by $\chi^{2}$ test, Kruskal-Wallis test, log-rank test, Cox proportional-hazards analysis, and the Cox score process. A $P$-value $<0.05$ was considered statistically significant. The statistical calculations were performed using $\mathrm{R}$ and SPSS Statistics 19.0.

\section{Results}

\section{The Histological Growth Patterns}

The pushing growth pattern was found in $33 \%$ of the metastases $(n=72)$, the desmoplastic pattern in $32 \%(n=70)$, the replacement pattern in $11 \%(n=23)$, and a mix of two growth patterns in $24 \%(n=52)$, as summarized in Table 1 . No metastases displayed all the three growth patterns. Among the mixed-type metastases, pushing growth was the most frequent pattern, appearing as the primary growth pattern in 
Table 1 Patient characteristics

Growth pattern

\begin{tabular}{|c|c|c|c|c|c|}
\hline \multirow[b]{2}{*}{ Variable } & & & & & \multirow[b]{2}{*}{ Total } \\
\hline & Replacement & Desmoplastic & Pushing & Mixed & \\
\hline Number of patients and (proportion) & $23(10.6 \%)$ & $70(32.3 \%)$ & $72(33.2 \%)$ & $52(23.9 \%)$ & $217(100 \%)$ \\
\hline Age, years $(P=0.2)^{\mathrm{a}}$ & $64(31-79)$ & $63(41-92)$ & $66(27-88)$ & $68(19-84)$ & $65(19-92)$ \\
\hline Sex ratio, $M / F(P>0.5)$ & $15 / 8$ & $41 / 29$ & $44 / 28$ & $34 / 18$ & $134 / 83$ \\
\hline Metastasis size, $c m(P=0.002)^{\mathrm{a}}$ & $2.4(0.5-16)$ & $1.9(0.4-15)$ & $2.9(0.6-30)$ & $2.8(0.3-11)$ & $2.5(0.3-30)$ \\
\hline Metastases per patient $(P>0.5)^{\mathrm{b}}$ & $2.0(1-7)$ & $2.0(1-5)$ & $1.8(1-5)$ & $2.0(1-9)$ & $1.9(1-9)$ \\
\hline Follow-up, months ${ }^{\mathrm{a}}$ & $22.8(2.7-50.3)$ & $31.1(1.8-76.3)$ & $32.8(0.4-74)$ & $29.7(0.2-76.3)$ & $30.1(0.2-76.3)$ \\
\hline Median survival, months & 22.8 & $>60$ & 44.2 & 40.3 & 44.2 \\
\hline
\end{tabular}

a Data expressed as median and (range).

${ }^{\mathrm{b}}$ Data expressed as mean and (range).

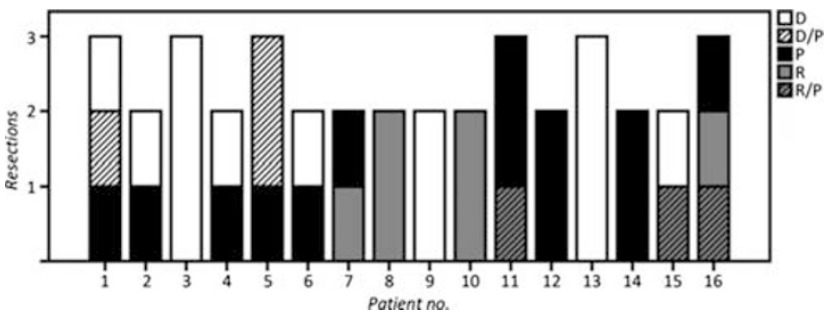

Figure 2 Diagram showing the growth pattern of colorectal liver metastases from patients with repeat hepatic resections (D, desmoplastic; P, pushing; R, replacement; in mixed-type patterns, the primary growth pattern is written first). In six cases, a recurrent metastasis displays a new growth pattern as compared with the patients' first metastasis. In seven cases, a recurrent metastasis shows a partly new growth pattern. Seven patients presents with only one pattern across their repeat resections.

$50 \%(n=26)$, and as the secondary growth pattern in $69 \%$ of the remaining $(n=18)$. Desmoplastic metastases were significantly smaller than metastases with pushing-type $(P=0.002$, Kruskal-Wallis test $)$ and mixed-type $(P=0.01)$ margins. No significant differences in size were found between metastases with desmoplastic and replacement growth or between metastases with replacement, mixed and pushing growth. The number of hepatic metastases per patient did not seem to vary across the patterns $(P>0.5$, Kruskal-Wallis test). However, these data were only available for 111 patients.

In addition, the growth pattern of 22 recurrent liver metastases was examined. Six of the recurrent metastases appeared with a new growth pattern as compared with the patients' first metastasis, and seven with a partly new growth pattern, in cases where one of the metastases showed a mixed-type margin. Consequently, the growth pattern does not seem to be host specific. The data are depicted in Figure 2.

\section{Survival Analysis}

Of the 217 patients included, 95 died during followup. Median survival was 44.2 months and median

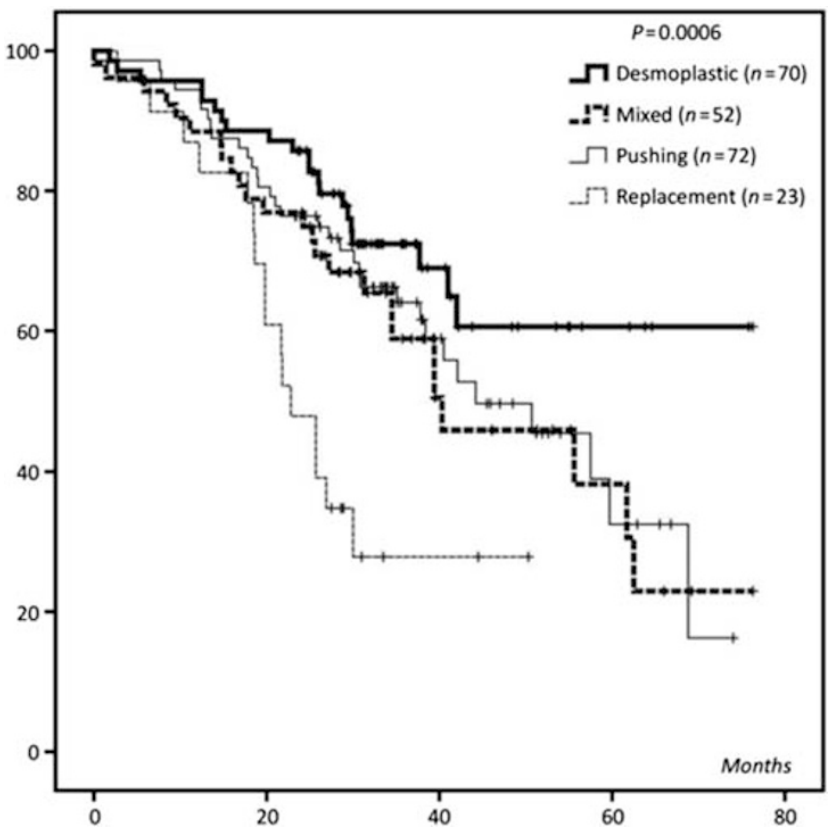

Figure 3 Kaplan-Meier plot showing the significance of the growth patterns in terms of overall survival $(n=217, P=0.0006)$. Replacement growth seems compatible with poor survival, whereas a desmoplastic growth pattern appears to be indicative of good survival. Patients with pushing-type and mixed-type metastases show intermediate survival rates.

follow-up time 30.1 months (range: 0.2-76.3). Kaplan-Meier analysis demonstrated significant differences in overall survival between patients with different growth patterns (Figure 3; $P=0.0006$, log-rank test). Patients with replacement growth appeared to have the poorest prognosis with a median survival of 22.8 months, as compared to 40.3 months in patients with mixed growth and 44.2 months in patients with pushing growth. Patients with desmoplastic metastases seemed to have the best prognosis with a five-year survival of $61 \%$ (median survival was not reached).

Cox analysis confirmed the replacement pattern as an independent predictor of poor survival 
Table 2 Cox proportional-hazards regression

\begin{tabular}{lcrr}
\hline & \multicolumn{2}{c}{ Unadjusted Cox regression } & \multicolumn{1}{c}{ Adjusted Cox regression $^{\mathrm{a}}$} \\
\cline { 2 - 3 } Variable & HR $(95 \%$ CI) & P-value & HR $(95 \%$ CI) \\
\hline Growth pattern & & & 1 \\
Replacement & 1 & $<0.0001$ & $0.21(0.11-0.42)$ \\
Desmoplastic & $0.27(0.14-0.51)$ & 0.004 & $0.32(0.18-0.61)$ \\
Pushing & $0.41(0.22-0.75)$ & 0.01 & $0.38(0.20-0.74)$ \\
Mixed & $0.45(0.24-0.85)$ & 0.001 & $1.07(1.02-1.12)$ \\
Tumor size, cm & $1.08(1.03-1.13)$ & 0.08 & - \\
Age, years & $1.02(1.00-1.04)$ & 0.25 & - \\
Sex, $0=F, 1=M$ & $1.28(0.84-1.95)$ & & 0.0001 \\
\hline
\end{tabular}

Abbreviations: CI, confidence interval; HR, hazard ratio.

${ }^{\mathrm{a}}$ Variables not in the equation: age $(P=0.5)$ and sex $(P=0.1)$.

(Table 2). For patients with replacement growth, the hazard of death was 2-2.5 times higher than for patients with pushing growth (hazard ratio (HR): $0.41,95 \%$ confidence interval (CI): 0.22-0.75, $P=0.004$ ) or mixed growth (HR: $0.45,95 \%$ CI: $0.24-0.85, P=0.01$ ), and nearly four times higher than for patients with desmoplastic growth (HR: 0.27, 95\% CI: $0.14-0.51, P<0.0001)$. The negative prognostic effect of a replacement-type margin was even more pronounced after adjusting for tumor size (Table 2). In the Cox analysis, desmoplastic growth was not associated with significantly better survival than pushing (HR: 1.53, 95\% CI: 0.89-2.65, $P=0.13$ ) and mixed growth (HR: 1.69, 95\% CI: 0.95-3.03, $P=0.08$ ); however, when matched against all nondesmoplastic metastases, the desmoplastic metastases showed a significant association with good survival (HR: 1.82, 95\% CI: 1.12-2.96, $P=0.015$ ). Patients with pushing- and mixed-type metastases did not differ with regard to survival (HR: 1.11, 95\% CI: $0.66-1.86, P>0.5)$. The Cox score process showed that the effects of growth pattern and tumor size on survival were independent of time, validating the assumption of proportional hazards. As the number of metastases per patient was only reported for 111 patients, this variable was not controlled for in the survival analysis.

\section{Immune Cell Infiltration}

Table 3 and Figure 4 show the density of the lymphocytic infiltrate at the tumor edge of 150 colorectal liver metastases grouped according to their primary growth pattern. As it appears, metastases with a desmoplastic growth pattern were significantly more densely infiltrated than both pushing-type $\left(P=0.0006, \chi^{2}\right.$ test $)$ and replacementtype $(P=0.0004)$ metastases. No significant difference was found between metastases with pushing and replacement growth $(P>0.5)$. Cox analysis showed no impact of lymphocytic infiltration on survival, neither univariately (HR: 0.78, 95\% CI:
Table 3 Density of the lymphocytic infiltrate

\begin{tabular}{lrrr}
\hline Primary growth pattern & \multicolumn{1}{c}{ Degree I } & Degree II & Degree III \\
\hline Replacement $(n=27)^{\mathrm{a}}$ & $10(37 \%)$ & $10(37 \%)$ & $7(26 \%)$ \\
Desmoplastic $(n=55)^{\mathrm{a}}$ & $3(5.5 \%)$ & $19(34.5 \%)$ & $33(60 \%)$ \\
Pushing $(n=68)^{\mathrm{a}}$ & $19(27.9 \%)$ & $28(41.2 \%)$ & $21(30.9 \%)$ \\
Total $(n=150)^{\mathrm{a}}$ & $32(21.3 \%)$ & $57(38 \%)$ & $61(40.7 \%)$
\end{tabular}

${ }^{\mathrm{a}}$ Data expressed as number and (proportion of $n$ ).

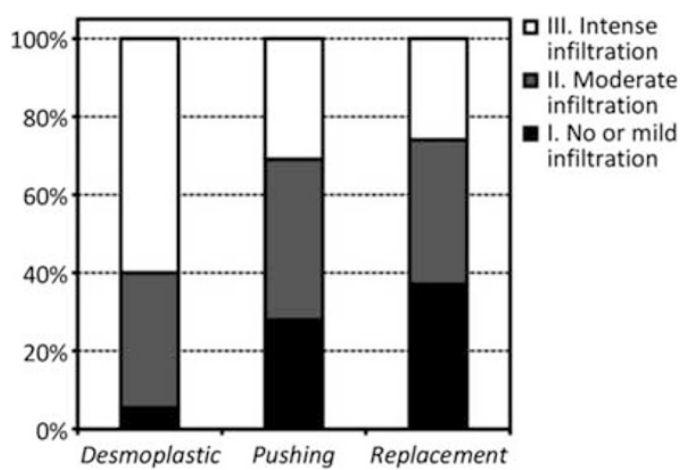

Figure 4 Histogram of the density of the immune cell infiltrate in relation to the primary growth pattern $(n=150)$. The desmoplastic metastases are significantly more heavily infiltrated than metastases with a pushing-type or replacement-type margin (desmoplastic vs pushing growth, $P=0.0006$; desmoplastic $v S$ replacement growth, $P=0.0004$; pushing $v s$ replacement growth, $P>0.5)$.

0.53-1.14, $P=0.2$ ), nor in a model adjusted for growth pattern and tumor size $(P>0.5)$.

\section{Discussion}

As compared to the primary colorectal adenocarcinomas, little is known on the pathogenesis and the prognostic factors of colorectal metastases in the 
liver. Vermeulen et al ${ }^{1}$ identified three histological growth patterns for colorectal hepatic metastases, indicating differences in the underlying pathobiology. As opposed to previous morphological characterizations of the tumor-liver parenchyma interface, Vermeulen's classification distinguishes three fairly homogenous groups, each defined positively by a distinctive set of histopathological characteristics. However, the clinical relevance and the biological background of the growth patterns remain to be determined. The findings of the current study revealed a prognostic significance of the growth patterns in terms of overall survival, showing a potential clinical applicability of the classification. Thus, a replacement-type margin was an independent predictor of poor survival, whereas a desmoplastic margin seemed compatible with a small tumor size and a more favorable prognosis. Eventually, the growth patterns may contribute to a histology-based prognostication of patients with colorectal metastases to the liver.

A few aspects of the current study deserve comments. Firstly, in the survival analysis, treatment before and after resection was not included in the model although, hypothetically, preoperative therapy may have affected the tumor morphology and treatment response may differ between the patterns. Hence, the survival data may not purely reflect the aggressiveness of the three types of expansion. However, from a clinical point of view, the findings are highly relevant, as the cohort reflects the actual patient population. Secondly, as the growth patterns were assessed on one tissue section per metastasis only, some of the observed growth patterns may not have been representative for the metastases. And thirdly, differences in the width of the resection margin could have influenced the results, although the prognostic value of this variable remains controversial.

The present findings conflict with a study conducted by Van den Eynden et $a l^{3}{ }^{3}$ which evaluated an older cohort of 205 patients hepatectomized for colorectal liver metastases in the period 19952005. In this study, no differences in overall survival were found across the growth patterns, yet pushing growth corresponded with poor 2-year survival. ${ }^{3}$ Although survival in all was markedly better in our study, we found a considerably poorer median survival for patients with replacement-type metastases than Van den Eynden et $a l^{3}{ }^{3}$ in spite of improved medical and surgical management. In addition, the prevalence of the replacement pattern was lower in our population. A possible explanation for these contrasting findings is the differences in treatment and selection of patients between the two studies, as the two populations are derived from different time periods. Thus, a large part of our patients had received adjuvant chemotherapy after resection of the primary tumor, or chemotherapy and targeted treatment neoadjuvantly in connection with their hepatic resection. In theory, this could have altered the growth pattern of the metastases, either through tumor regression with subsequent scar tissue formation or through a selection of chemoresistant tumor clones with specific growth characteristics. Furthermore, different treatment response among the patients could have impacted on the results; in particular, anti-angiogenic therapy may have a stronger effect on pushing-type tumors than on replacement-type tumors. In both studies, desmoplastic encapsulation appeared as a marker of favorable outcome. ${ }^{3}$

The findings obtained in the current study indicate a particularly aggressive or treatment resistant tumor biology of replacement-type metastases. One reason for this could be that metastases with a replacement-type margin gain an advantage by incorporating supporting structures endogenous to the liver. Pushing-type and desmoplastic metastases, on the other hand, depend entirely on the formation of new blood vessels and structural stroma gradually as they expand; the high angiogenic activity is, at least in part, hypoxia-driven. ${ }^{3}$ Correlations between low-angiogenic growth patterns and poor survival have also been reported for primary non-small-cell lung cancer. ${ }^{14,15}$ In addition to this, hypothetically, the intercellular interactions between tumor cells and hepatocytes in the replacement pattern (see Figure 1d) may exert tumor-promoting properties. It should be emphasized that the intermediate survival rates for patients with pushing- and mixed-type metastases could reflect heterogeneity within these two groups with both highly aggressive and more indolent tumors represented.

As regard the desmoplastic pattern, the question is, whether the tumor actively stimulates fibrogenesis in order to generate a favorable microenvironment or whether the stromal reaction rather represents a hepatic defense mechanism, an attempt to confine the tumor, analogous to a host-versusgraft response. It is well described that desmoplastic encapsulation of colorectal liver metastases corresponds with good survival, a minimal risk of local recurrence, and small tumor size..$^{3,7-13}$ It has even been reported that the thicker the desmoplastic rim is, the better the prognosis. ${ }^{11,12}$ These data corroborate that the desmoplastic pattern might teleologically represent a hepatic defense mechanism, at least as the general rule. Indeed, the cellular and molecular mechanisms involved in encapsulation of hepatic tumors resemble those of wound healing in response to chronic liver injury. ${ }^{16-20}$ However, for some cell lines from colorectal hepatic metastases, in vitro interactions with type-I collagen have been reported to promote growth, survival, and chemoresistance via $\alpha_{\mathrm{v}}$ integrin ligation. ${ }^{21}$

Vermeulen et $a l^{1}$ described the growth patterns as three fundamentally different types of expansion, yet the mixed-type pattern and the variable growth patterns among multiple and recurrent metastases, documented by Eefsen et $a l^{4}$ and the present study, 
suggest interrelations between the growth patterns. Based on a study on autopsied livers, which showed that the replacement pattern was highly predominant among micrometastases $<1 \mathrm{~mm}$, Terayama et $a l^{22}$ hypothesized that the replacement pattern is simply a stage that precedes the pushing and desmoplastic patterns. As the metastasis progresses, the morphological appearance of the tumor-liver boundary will change either with the accumulation of collagen or when the speed of expansion exceeds the rate of hepatocyte replacement at the invasive front. $^{22}$ Terayama's notion is not necessarily incompatible with the relationship between replacement growth and poor survival, demonstrated in the present study. Hence, the current findings could also indicate that metastases, which continue to grow according to a replacement pattern, in general are the most aggressive.

In murine models, Paku et $a l^{23}$ demonstrated that slow growth rate favor the accumulation of fibrous tissue, in particular in response to pressure atrophy due to pushing growth. In consequence, the reason why pushing growth is overrepresented among mixed-type metastases could be that replacementtype metastases with slow hepatocyte turnover tend to progress to the pushing pattern, whereas slowly expanding pushing-type tumors are particularly likely to induce a stromal reaction, leading to a desmoplastic pattern.

In conclusion, the present study demonstrated a prognostic value of the growth patterns of colorectal liver metastases in terms of overall survival. If the current findings can be reproduced, we recommend recording the growth pattern as a routine prognostic biomarker in the pathology report. In addition, the growth patterns may in time be useful in the stratification of patients for personalized medicine, although the predictive potential would improve if biochemical or radiographic surrogates were discovered. The prognostic impact of the classification reinforces the need for identifying the factors that determine the growth pattern. A possible determinant was described by a recent study, which categorized colorectal cancer cells according to their expression of Wnt-target and stem cell genes, identifying six subtypes, each resembling a distinct cell type within the normal colon crypt. ${ }^{24}$ These subtypes could be indicative of the development of metastatic disease and may correspond with the morphology of the metastatic tumors.

\section{Acknowledgments}

The work was supported financially by the following councils and foundations: Rigshospitalet's Research Grants; PA Messerschmidt and Hustrus Foundation; The Danish Medical Association's Research Foundation; and Aage Thuesen Bruun and Hustru Emmy Katy Bruun's Commemoration Grant.

\section{Disclosure/conflict of interest}

The authors declare no conflict of interest.

\section{References}

1 Vermeulen PB, Colpaert C, Salgado R, et al. Liver metastases from colorectal adenocarcinomas grow in three patterns with different angiogenesis and desmoplasia. J Pathol 2001;195:336-342.

2 Stessels F, van den Eynden G, van der Auwera I, et al. Breast adenocarcinoma liver metastases, in contrast to colorectal cancer liver metastases, display a nonangiogenic growth pattern that preserves the stroma and lacks hypoxia. Br J Cancer 2004;90:1429-1436.

3 Van den Eynden GG, Bird NC, Majeed AW, et al. The histological growth pattern of colorectal cancer liver metastases has prognostic value. Clin Exp Metastasis 2012;29:541-549.

4 Eefsen RL, Van den Eynden GG, Høyer-Hansen G, et al. Histopathological growth pattern, proteolysis and angiogenesis in chemonaive patients resected for multiple colorectal liver metastases. J Oncol 2012; 2012:907971.

5 Illemann M, Bird N, Majeed A, et al. Two distinct expression patterns of urokinase, urokinase receptor and plasminogen activator inhibitor-1 in colon cancer liver metastases. Int J Cancer 2009;124:1860-1870.

6 Nagashima I, Oka T, Hamada C, et al. Histopathological prognostic factors influencing long-term prognosis after surgical resection for hepatic metastases from colorectal cancer. Am J Gastroenterol 1999;94:739-743.

7 Ambiru S, Miyazaki M, Isono T, et al. Hepatic resection for colorectal metastases: analysis of prognostic factors. Dis Colon Rectum 1999;42:632-639.

8 Lunevicius R, Nakanishi H, Ito S, et al. Clinicopathological significance of fibrotic capsule formation around liver metastasis from colorectal cancer. J Cancer Res Clin Oncol 2001;127:193-199.

9 Morino T, Tanaka J, Tobe T. Clinico-pathological features of liver metastases from colorectal cancer in relation to prognosis. Nihon Geka Hokan 1991;60:154-164.

10 Ohlsson B, Stenram U, Tranberg KG. Resection of colorectal liver metastases: 25-year experience. World J Surg 1998;22:268-276.

11 Okano K, Yamamoto J, Kosuge T, et al. Fibrous pseudocapsule of metastatic liver tumors from colorectal carcinoma.Clinicopathologic study of 152 first resection cases. Cancer 2000;89:267-275.

12 Weber JC, Nakano H, Bachellier P, et al. Is a proliferation index of cancer cells a reliable prognostic factor after hepatectomy in patients with colorectal liver metastases? Am J Surg 2001;182:81-88.

13 Yamamoto J, Shimada K, Kosuge T, et al. Factors influencing survival of patients undergoing hepatectomy for colorectal metastases. Br J Surg 1999;86: 332-337.

14 Sardari Nia P, Colpaert C, Blyweert B, et al. Prognostic value of nonangiogenic and angiogenic growth patterns in non-small-cell lung cancer. Br J Cancer 2004;91:1293-1300.

15 Sardari Nia P, Van Marck E, Weyler J, et al. Prognostic value of a biologic classification of non-small-cell lung cancer into the growth patterns along with other clinical, pathological and immunohistochemical factors. Eur J Cardiothorac Surg 2010;38:628-636. 
16 Hautekeete ML, Geerts A. The hepatic stellate (Ito) cell: its role in human liver disease. Virchows Arch 1997;430:195-207.

17 Li D, Friedman SL. Liver fibrogenesis and the role of hepatic stellate cells: new insights and prospects for therapy. J Gastroenterol Hepatol 1999;14:618-633.

18 Théret N, Musso O, Turlin B, et al. Increased extracellular matrix remodeling is associated with tumor progression in human hepatocellular carcinomas. Hepatology 2001;34:82-88.

19 Bissell DM. Chronic liver injury, TGF-beta, and cancer. Exp Mol Med 2001;33:179-190.

20 Friedman SL. Mechanisms of hepatic fibrogenesis. Gastroenterology 2008;134:1655-1669.
21 Conti JA, Kendall TJ, Bateman A, et al. The desmoplastic reaction surrounding hepatic colorectal adenocarcinoma metastases aids tumor growth and survival via $\alpha_{v}$ integrin ligation. Clin Cancer Res 2008;14:6405-6413.

22 Terayama N, Terada T, Nakanuma Y. Histologic growth patterns of metastatic carcinomas of the liver. Jpn J Clin Oncol 1996;26:24-29.

23 Paku S, Kopper L, Nagy P. Development of the vasculature in "pushing-type" liver metastases of an experimental colorectal cancer. Int J Cancer 2005;115:893-902.

24 Sadanandam A, Lyssiotis CA, Homicsko K, et al. A colorectal cancer classification system that associates cellular phenotype and responses to therapy. Nat Med 2013;19:619-625. 